DSF-22-2007

\title{
HIGH ENERGY NEUTRINO SIGNALS FROM THE EPOCH OF REIONIZATION
}

\author{
F. Iocco ${ }^{1,2}, \mathrm{~K} . \mathrm{Murase}^{3}, \mathrm{~S} . \mathrm{Nagataki}^{2,3}, \mathrm{P}$. D. Serpico ${ }^{4}$ \\ ${ }^{1}$ Università di Napoli "Federico II", Dip. Scienze Fisiche, via Cintia, 80126 Napoli, Italy \\ ${ }^{2}$ Kavli Institute for Particle Astrophysics and Cosmology PO Box 20450, Stanford, CA 94309, USA \\ ${ }^{3}$ YITP, Kyoto University, Kyoto, Oiwake-cho, Kitashirakawa, Sakyo-ku, Kyoto, 606-8502, Japan \\ ${ }^{4}$ Center for Particle Astrophysics, Fermi National Accelerator Laboratory, Batavia, IL 60510-0500 USA \\ Draft version July 3, 2007
}

\section{ABSTRACT}

In this paper we perform a new estimate of the high energy neutrinos expected from GRBs associated with the first generation of stars in light of new models and constraints on the epoch of reionization and a more detailed evaluation of the neutrino emission yields. We also compare the diffuse high energy neutrino background from Population III stars with the one from "ordinary stars" (Population II), as estimated consistently within the same cosmological and astrophysical assumptions. In disagreement with previous literature, we find that high energy neutrinos from Population III stars will not be observable with current or near future neutrino telescopes, falling below both IceCube sensitivity and atmospheric neutrino background under the most extreme assumptions for the GRB rate. This rules them out as a viable diagnostic tool for these still elusive metal-free stars.

Subject headings: stars: early-type - gamma rays: bursts, neutrinos - cosmology: theory

\section{INTRODUCTION}

The first generation of stars (Population III or PopIII stars) born after the collapse of the very first structures in the universe is puzzling the astrophysical community since long time. The vanishing metallicity of the universe at the epoch of their formation (Iocco et al. '07) is supposed to give them peculiar properties, all arising from their very high characteristic mass due to the peculiar cooling of the cloud (Abel et al. '01). In fact, stars of $\mathcal{O}(100) M_{\odot}$ have very short lifetimes and either explode as pair instability Super Novae (PISNe) or directly collapse to black holes (Heger et al. '03). They also contribute to the metallicity enrichment of the early universe, shaping the transition to the second generation of stars. Moreover, the very high temperature of these objects makes them efficient engines for the production of Lyman-Werner ultraviolet photons, thus initiating the cosmic reionization process. Unfortunately, all of the traces PopIII stars leave behind are challenging to observe and so far no unambiguous detection has been recognized, although the detection of anisotropies in the infrared background consistent with the existence of PopIII has been claimed (Kashlinsky et al. '05). Qualitatively, their peculiar initial mass function (IMF) should increase their Super Nova (SN) rate with respect to later stellar populations. A fairly general expectation is that PopIII may emit large amounts of neutrinos, both thermally produced at the time of the collapse (Iocco et al. '05) and non-thermally during a GammaRay Burst (GRB) phase associated with the explosion (Schneider et al. '05). GRBs are one of the candidate hadronic accelerators, and any proton accelerator is a potential emitter of high energy neutrinos, produced via hadronic $(p p)$ or photo-hadronic $(p \gamma)$ reactions in the surrounding medium. Although the details of this whole picture are far from being established and despite the large uncertainties in the models, the idea of using neutrinos to probe PopIII stars surely deserves further study.

In this paper we perform a new estimate of the high en- ergy neutrinos expected from GRBs associated with the first generation of stars in light of new models and constraints on the epoch of reionization (EoR) and a more detailed evaluation of the neutrino emission yields. The goal of this paper is two-fold: $(i)$ to compare the diffuse high energy neutrino background from PopIII with the one from "ordinary" (PopII) stars that contribute with PopIII to the reionization at $7 \lesssim z \lesssim 12 ;$ (ii) to discuss the chances of detection on the light of the performances expected for current or future neutrino telescopes as a function of the astrophysical input. Indeed both conditions need to be fulfilled to establish if there is any realistic chance to discriminate the high energy neutrino emission from PopIII. To do so, after introducing the basic formalism in Sec. 2. we shall preliminarily discuss what a semi-empirical estimate of the GRB rate at high redshift can tell us (Sec. 3). Then, we devote Sec. 4 to describe the models used to perform theoretical estimates of the neutrino fluxes. We shall pay particular attention to compare estimates derived consistently within the same cosmological assumptions. In Sec. 5 we present our results and compare with the chances of detection, and finally in Sec. 6 we conclude. In Appendix A we report some detail of the GRB model used to compute the neutrino yields.

\section{BASIC FORMALISM}

In this Section we establish the formalism we will use throughout the paper to estimate the diffuse flux of neutrinos emitted by GRBs. The integrated signal observed today at energy $E_{\nu}$ is

$$
\begin{array}{r}
E_{\nu}^{2} \Phi_{\nu}\left[\mathrm{GeV} \mathrm{cm}^{-2} \mathrm{~s}^{-1} \mathrm{sr}^{-1}\right] \equiv E_{\nu}^{2} \frac{\mathrm{d} F_{\nu}}{\mathrm{d} E_{\nu}}= \\
\int \mathrm{d} z \frac{\mathrm{d} \dot{N}_{\mathrm{G}}(z)}{\mathrm{d} z} \frac{\mathrm{d} N_{\nu}^{\text {iso }}}{\mathrm{d} E_{\nu} \mathrm{d} A}\left(E_{\nu}, z\right),
\end{array}
$$

where $\mathrm{d} \dot{N}_{\mathrm{G}} / \mathrm{d} z$ is the differential rate of GRBs which beam towards us, and $\mathrm{d} N_{\nu}^{\text {iso }} / \mathrm{d} E_{\nu} \mathrm{d} A$ is the average flux emitted by a single source at energy $E_{\nu}(1+z)$. 
The function $\mathrm{d} \dot{N}_{\mathrm{G}} / \mathrm{d} z$ can be written as

$$
\frac{\mathrm{d} \dot{N}_{\mathrm{G}}}{\mathrm{d} z}(z)=\frac{\rho_{\mathrm{G}}(z)}{(1+z)} \frac{\mathrm{d} V}{\mathrm{~d} z}=\frac{b G(z)}{(1+z)} \frac{\mathrm{d} V}{\mathrm{~d} z},
$$

where $\rho_{\mathrm{G}}(z)$ is the comoving GRB rate in unit of the proper volume and $\mathrm{d} V$ is the comoving volume element, such that

$$
\frac{\mathrm{d} V}{\mathrm{~d} z}=4 \pi \frac{c}{H(z)} r^{2}(z) .
$$

We have introduced the comoving distance $r(z)$ defined as

$$
r(z)=\int_{0}^{z} \frac{c}{H(w)} \mathrm{d} w .
$$

The Hubble function $H(z)$ writes in terms of the fractions of the critical energy density in matter $\Omega_{M}$ and cosmological constant $\Omega_{\Lambda}$ as

$$
H(z)=H_{0} \sqrt{\Omega_{M}(1+z)^{3}+\Omega_{k}(1+z)^{2}+\Omega_{\Lambda}},
$$

$H_{0}$ being the inverse Hubble distance today $H_{0}^{-1} \simeq$ $3 h^{-1} \mathrm{Gpc}$ and $\Omega_{k}=1-\Omega_{M}-\Omega_{\Lambda}$. We have also denoted by $b=\left\langle 1-\cos \theta_{\text {jet }}\right\rangle$ the averaged beaming factor of a jet of opening angle $\theta_{\text {jet }}$, so that $G(z)$ is the beaming-corrected overall GRB rate. This is also the ratio between the physical energy $E_{\text {jet }}$ and the one released by the GRB if that was isotropic, $b=E_{\text {jet }} / E_{\text {iso. }}$. If we introduce the function $J_{\nu}$ of the energy at the source $E_{\nu}^{\prime}$

$$
J_{\nu}\left[E_{\nu}^{\prime}\right] \equiv E_{\nu}^{\prime 2} \frac{\mathrm{d} N_{\nu}^{\text {iso }}}{\mathrm{d} E_{\nu}^{\prime}}
$$

the average energy flux, $E_{\nu}^{2}\left(\mathrm{~d} N_{\nu}^{\text {iso }} / \mathrm{d} E_{\nu} \mathrm{d} A\right)$ (units $\mathrm{GeV}$ $\mathrm{cm}^{-2}$ ), observed at a given energy $E_{\nu}$ and emitted by a source at redshift $z$ can be expressed as

$$
E_{\nu}^{2} \frac{\mathrm{d} N_{\nu}^{\text {iso }}}{\mathrm{d} E_{\nu} \mathrm{d} A}\left(E_{\nu}, z\right)=\frac{1}{1+z} \frac{J_{\nu}\left[E_{\nu}(1+z)\right]}{4 \pi r^{2}(z)} .
$$

In the previous expression, $E_{\nu}(1+z)$ is the emission energy, $J_{\nu}\left[E_{\nu}(1+z)\right] /(1+z)$ takes into account the redshifted energy spectrum, and possible boosting factors are already included in $J_{\nu}$.

Putting the two pieces together, we finally get

$$
E_{\nu}^{2} \Phi_{\nu}=\frac{c b}{4 \pi} \int \mathrm{d} z \frac{J_{\nu}[E(1+z)] G(z)}{(1+z)^{2} H(z)} .
$$

It remains to estimate $G(z)$ and $J_{\nu}(E)$, a problem which we shall address in the following.

\section{AN EMPIRICAL ESTIMATE OF GRB RATE}

Let us start by using an empirical approach: the GRB rate is derived from observations and extrapolated at high redshifts, as in (Yonetoku et al. '03) and (Fenimore \& Ramirez-Ruiz '05). This approach assumes the $E_{p}$-luminosity relation, (Amati et al. '02), to infer the redshift of a GRB whose host is at unknown redshift. Note that in order to extrapolate the $E_{p}$-luminosity relation to high redshifts, one implicitly assumes that although GRBs at high redshift might belong to different stellar populations with different star formation rates (SFRs) and IMFs, they constitute "standard candles" carrying no memory of the population of the progenitors they belong to.
Aware of this caveat, the most natural hint of a transition PopIII-PopII would then be a break of the power-law dependence of the GRB formation history on the redshift; indeed, one should expect in general that the conditions for the GRB appearance will occur at a different rate in stellar populations with different characteristics. This is manifestly not the case of the GRB rate derived empirically from observations in (Yonetoku et al. '03) and (Fenimore \& Ramirez-Ruiz '05): no discontinuity appears at $6<z<12$, where a PopIII-PopII transition would suggest to see it. In particular, these results indicate that the GRB formation rate always increases toward $z \sim 12$, in a way parameterized by:

$$
\rho_{\mathrm{emp}}(z)=\rho_{\mathrm{emp}}(0) \times \begin{cases}(1+z)^{\alpha}, & z<1 \\ 2^{\alpha-\beta}(1+z)^{\beta}, & 1<z \lesssim 12,\end{cases}
$$

where $\alpha=6.0 \pm 1.4, \beta=0.4 \pm 0.2$ and

$$
\rho_{\mathrm{emp}}(0)=2.3 \times 10^{-14} \mathrm{~s}^{-1} \mathrm{Mpc}^{-3},
$$

all the quantities being fixed according to (Murakami et al. '05), where for the normalization we use their intermediate estimate for the luminosity correction $(k=0.5)$. Note that if we assume a simple top-hat function for $J_{\nu}$, from Eq. (8) and the fit for $\rho_{\mathrm{emp}}(z)$ it is easy to check that neutrinos emitted at high redshift have at most a subleading contribution to the total flux. Qualitatively, this is consistent with what found e.g. in (Murase \& Nagataki '05): most of the GRB neutrino signal is not sensitive to the GRB rate at high redshift.

This general result might be interpreted as (weak) empirical evidence for the dominance of PopII progenitors in the overall GRB population at any redshift. However, this conclusion only holds under two assumptions: $(i)$ the validity of the $E_{p}$-luminosity relation up to high redshifts; since this is an empirical relation and the engine of GRB is far from being understood, there is no guarantee that this is the case; (ii) the absence of a significant fraction of choked GRBs among PopIII stars. It has been argued that due to the different structure and properties of PopIII stars the jet in a collapsar may be unable in most cases to punch through the stellar envelope (MacFadven et al. '01), thus choking the burst in gamma rays, although still producing large neutrino yields. This was also the working hypothesis of (Schneider et al. '05). These arguments suggest that a more realistic estimate of the neutrino flux from PopIII GRB may require some degree of modeling, which we are going to address in the next section.

\section{THEORETICAL ESTIMATES OF THE NEUTRINO FLUX}

Let us now estimate the GRB rate at high redshifts from current theoretical models on the stellar populations at high redshift, under the assumption that the GRB rate tracks the SFR. Besides the SFR for the different stellar populations, it is clearly mandatory to know their IMFs in order to evaluate the fraction of stars that are likely to end their lives as collapsars, believed to give rise to a GRB. In this section we present the neutrino fluxes expected from the two different populations of stars under different EoR models available in literature. The three models of reionization we consider are summarized in Table [1 the "fiducial" model presented in (Choudhury \& Ferrara '05), figure 1 


\begin{tabular}{|l|l|l|}
\hline Model & Reference & Notes \\
\hline \hline CF05 $a$ & (Choudhury \& Ferrara '05) & high ion. efficiency \\
\hline CF05 $b$ & (Choudhury \& Ferrara '05) & low ion. efficiency \\
\hline CF06 & (Choudhury \& Ferrara '06) & WMAP3, $S(M)$ \\
\hline
\end{tabular}

TABLE 1

THE MODELS OF REIONIZATION WE CONSIDER FOR CALCULATING THE NEUtrino FluXes. SeE TEXT FOR DEtails.

at page 586 (hereafter $\mathrm{CF} 05_{a}$ ); the one in figure 4, page 590 of the same paper (hereafter $\mathrm{CF} 05_{b}$ ); the model in (Choudhury \& Ferrara '06) (hereafter CF06), which takes into account the new cosmological data on reionization from the third-year data release of the WMAP team (Spergel et al. '06). These models are used in the following to derive the star formation rates needed for our estimates. All the CF-models share the same physical assumptions: three radiation sources are taken into account during EoR: Quasi Stellar Objects (QSOs), PopII, and PopIII stars which contribute with different characteristics to the radiative and chemical enrichment of the IGM, and to the consequent feedback. Among the many parameters of the models, a key role is played by the fraction of escaping photons per halo (directly related to the ionizing efficiency), which must be deduced from the knowledge of the source and halo characteristics of PopII and PopIII stars; the luminosity function of QSOs is calculated according to standard assumptions. Both the CF05 models assume that all PopIII stars have a mass of $300 \mathrm{M}_{\odot}$ while PopII follow a Salpeter IMF, $S(m)$. It is also worth mentioning that the transition between PopIII and PopII stars, regulated by the chemical feedback, is considered to be instantaneous. The only difference between $\mathrm{CF} 05_{a}$ ad $\mathrm{CF} 05_{b}$ is the ionizing efficiency of PopIII, which in the latter model is assumed to be $2 / 7$ of the former one. This drastically changes the normalization and shape of the PopIII SFR. With respect to the CF05 models, in CF06 the stellar formation in minihaloes is suppressed, and a self-consistent calculation is made of the chemical enrichment process, which takes longer and results in a non-instantaneous transition between PopII and PopIII. For our purposes, however, the crucial difference is that CF06 assumes a Salpeter IMF for the PopIII, too. The authors implement this choice in order to match the NICMOS high redshift source counts. We address the reader to the original papers for more details. Also note that none of the models provides the low-redshift $(z \lesssim 3)$ extrapolation of the SFR. Therefore, for the neutrino fluxes from PopII shown as comparison in the following, we have used at low-redshift the GRB rate given in Eq. (12c) of (Murase \& Nagataki '05) normalized in order to match the observe GRB rate $z=0$.

\subsection{Rate of GRBs from PopII}

If we denote by $R_{\mathrm{II}}$ the SFR of PopII stars (taken from the models reported in Table 1), an estimate for the number density rate of GRBs from PopII stars, $G_{\mathrm{II}}$, can be obtained as

$$
G_{\mathrm{II}}(z)=\sigma_{\mathrm{II}} \gamma_{\mathrm{II}} \frac{R_{\mathrm{II}}(z)}{M_{\mathrm{II}}}
$$

where $M_{\text {II }}$ is the average mass of a PopII star, $\sigma_{\text {II }}$ is the fraction of PopII which are likely to form core collapse
Supernovae and $\gamma_{\mathrm{II}}$ is the fraction of core collapse SuperNovae which are likely to form a GRB. The average mass of a PopII star is obtained via an integral over their IMF, $\mathcal{I}_{\text {II }}$, as

$$
M_{\text {II }}=\frac{\int \mathcal{I}_{\text {II }}(M) M \mathrm{~d} M}{\int \mathcal{I}_{\text {II }}(M) \mathrm{d} M},
$$

assuming (consistently with all the EoR models considered) that $\mathcal{I}_{\mathrm{II}}(M)$ is a Salpeter mass function $S(M)$ which writes (Kroupa '00)

$$
S(m) \propto m^{-\alpha_{i}} \begin{cases}\alpha_{0}=0.3, & 0.01<m<0.08 \\ \alpha_{1}=1.3, & 0.08<m<0.50 \\ \alpha_{2}=2.3, & 0.50<m,\end{cases}
$$

where $m$ is the mass in solar units $M_{\odot}$. This is also used to estimate $\sigma_{\mathrm{II}}$, assuming that all PopII with $m>10$ will end their lives as SN,

$$
\sigma_{\mathrm{II}} \simeq \frac{\int_{10}^{125} S(m) \mathrm{d} m}{\int_{0.1}^{125} S(m) \mathrm{d} m} .
$$

We allow for a $z$-dependence in $\gamma_{\mathrm{II}}$ to take into account the GRB-metallicity anti-correlation and the fact that the metallicity evolves with the age of the Universe. Following (Yuksel \& Kistler '06) and (Yoon et al. '06),

$$
\gamma_{\mathrm{II}}(z)=\gamma_{\mathrm{II}}(0)(1+z)^{1.4}=\frac{(1+z)^{1.4}}{1250}
$$

where we fixed $\gamma_{\mathrm{II}}(0)$ according to (Yoon et al. '06) and $\gamma_{\mathrm{II}}(z)$ has been considered constant for $z>4.5$, thus considering no metallicity evolution above this redshift (however, this choice affects very little the final results, most of the contributions to the GRB neutrino background coming from bursts that occur at $z \lesssim 7$ ).

\subsection{Rate of GRB from PopIII}

Formally, the number density rate of GRB from PopIII stars, $G_{\text {III }}$ can be written identically to Eq. (11), namely

$$
G_{\mathrm{III}}(z)=\sigma_{\mathrm{III}} \gamma_{\mathrm{III}} \frac{R_{\mathrm{III}}(z)}{M_{\mathrm{III}}},
$$

where the various coefficients and functions involved now assume different values. The $R_{\mathrm{III}}(z)$ are taken from the models reported in Table 1 Concerning the IMF of PopIII stars, $\mathcal{I}_{\text {III }}$, there are currently two hypotheses: $(i)$ a bimodal IMF with a first peak at a few solar masses and the second one at $\sim 100 M_{\odot}$ (Nakamura \& Umemura '01); (ii) a peaked IMF around the range of few hundred solar masses has been later presented in (Abel et al. '01). Recently it has been pointed out, (O'Shea et al. '05), that primordial stars forming in a halo neighboring the formation star of a PopIII have still zero metallicity but smaller accretion rates onto the central dense region, resulting in a final stellar mass of $10-100 \mathrm{M}_{\odot}$. This "intermediate" generation would have equally contributed to the reionization and we do implement this hypothesis by studying the contribution of $M \sim 60 M_{\odot}$ in our neutrino models. Whatever the details are, the hypothesis $(i i)$ is nowadays widely accepted in the community, or in any case it is the $\mathcal{I}_{\text {III }}$ most frequently implemented in EoR models. We shall consider the following cases: 


\begin{tabular}{|c|c|c|c|c|c|}
\hline Model & Mass $\left(M_{\odot}\right)$ & $E_{\text {iso }}(\mathrm{erg})$ & $E_{\text {jet }}(\mathrm{erg})$ & $r_{\text {is }}(\mathrm{cm})$ & $\eta$ \\
\hline \hline Hidden-A & 200 & $10^{54}$ & $10^{52}$ & $10^{13.0}$ & 1 \\
\hline HIdden-B & 200 & $10^{54}$ & $10^{51}$ & $10^{13.0}$ & 1 \\
\hline Hidden-C & 60 & $10^{53}$ & $10^{51}$ & $10^{12.5}$ & 1 \\
\hline Hidden-D & 60 & $10^{53}$ & $10^{50}$ & $10^{12.5}$ & 1 \\
\hline Hidden-E & 200 & $10^{54}$ & $10^{52}$ & $10^{11.0}$ & 10 \\
\hline Hidden-F & 60 & $10^{53}$ & $10^{51}$ & $10^{11.5}$ & 10 \\
\hline \hline Prompt & $35-125$ & $10^{53}$ & $1.24 \times 10^{51}$ & $10^{13-15.5}$ & 1 \\
\hline
\end{tabular}

TABLE 2

The PARAMETERS VARIED IN THE MODELS OF GRB CONSIDERED.

$(a)$

$$
\mathcal{I}_{\mathrm{III}} \propto \delta\left(M_{\mathrm{III}}-300 M_{\odot}\right),
$$

i.e. a toy model of a "monochromatic" high mass mode for the IMF.

(b)

$$
\mathcal{I}_{\text {III }} \propto \delta\left(M_{\text {III }}-60 M_{\odot}\right),
$$

i.e. a toy model of a monochromatic IMF, with the average mass of metal-free stars forming from the collapse of halos triggered by the explosion of the very first stars.

$(c)$

$$
\mathcal{I}_{\text {III }} \propto S(M),
$$

as assumed e.g. in (Choudhury \& Ferrara '06).

Note that in both case $(a)$ and case $(b)$ one has $\sigma_{\text {III }} \simeq 1$, while in case $(c)$ one obtains an expression analogous to Eq. (14).

Concerning $\gamma_{\mathrm{III}}$, there are no firm estimates. For the sake of clarity and to simplify the comparison with previous results of (Schneider et al. '05), we shall assume the same value,

$$
\gamma_{\mathrm{III}} \simeq 1
$$

This corresponds to an absolute upper limit to the PopIII GRBs; a smaller GRB efficiency would result in a downscaling of the results presented in the following.

\subsection{Neutrino yields from GRBs}

High energy neutrinos from GRBs are predicted in various scenarios. The one most frequently discussed is neutrino emission in the internal shocks that produce prompt emission (Waxman \& Bahcall '97). In this scenario, accelerated protons interact with gamma-rays via photomeson production, and produce pions and kaons, which decay into neutrinos. The observed gammaray spectra are usually represented by a broken powerlaw. As for proton spectra, a first order Fermi-type spectrum is frequently assumed. This possibility has been studied by many authors. Murase \& Nagataki (Murase \& Nagataki '05); Murase \& Nagataki '06) did such calcultions using Geant4 with experimental data. They also took into account various cooling processes. One parameter set of their results (which is used in Achterberg et al. (Achterberg et al. '07)) will be shown for comparison in this paper, and named Prompt in the following.

Another model was first suggested by Mészáros and Waxman (Meszaros \& Waxman '01) and later extended in (Razzaque et al. '03). The GRB progenitor is usually taken to be a massive star with a He core and $\mathrm{H}$ envelope. The central engine of GRBs is still a major open problem in astrophysics; the leading model for (long duration) GRBs is a huge stellar collapse leading to formation of a highly rotating black hole with an accretion disk (collapsar model). The jet which produces succeeded GRBs or choked GRBs propagate in stars. This jet would interact with the stellar envelope before it produces prompt emission. In addition, the observed submillisecond variability allows us to expect that intenal shocks can occur at sufficiently small radii, where the Thomson optical depth exceeds unity. Protons can be accelerated in such internal shocks, and they can interact with photons from electrons that are accelerated in internal shocks and/or termination shocks. Photons from such inner radii cannot escape due to large optical thickness, so that they are hidden sources as gamma-ray sources. Only neutrinos would be useful as the probe of physical processes in this region. Since here we want to estimate the high energy neutrinos from PopIII GRBs, consistently with the empirical suggestion from Sec. 3 we shall only evaluate neutrino emission from such inner radii. Indeed, this emission is present also for chocked GRBs. Throughout the paper, we take into account neutrino oscillations in vacuum by adopting mixing angles $\theta_{12}=0.59, \theta_{23}=\pi / 4$, and $\theta_{13}=0$. The yields shown in the rest of this paper refer to the muon neutrino flavor, which is the best channel for detection at neutrino telescopes.

Since our knowledge of the GRB engine and PopIII properties is still limited, it is unclear how efficient are PopIII stars as GRB progenitors. But rotation and chemical mixing seem to be a key ingredient towards successful collpsar models (Maeder \& Mevnet '00; Heger et al. '00), and recent simulations in (Yoon et al. '06) suggest that GRBs can be born from PopIII stars, an hypothesis we will assume throughout. We expect that more massive star collapses lead to larger released energies (Heger et al. '03). Observationally, to ease the energy requirements, it is usually considered that a globally asymmetric, relativistic jet is launched from GRB progenitors. In fact, this picture is suggested by some observations, e.g. (Frail et al. '01). The jet energy is typically $E_{\text {jet }} \sim 1.24 \times 10^{51} \mathrm{erg}$. However, the Ghirlanda relation (Ghirlanda et al. '04) implies that bursts can have various jet energies, and some GRBs may have a jet energy $E_{\text {jet }} \sim 10^{52} \mathrm{erg}$, which is larger than the frequently used value $E_{\text {jet }} \sim 1.24 \times 10^{51}$ erg. The isotropic energy is highly uncertain, too, and it may be as high as $E_{\text {iso }} \sim 10^{54} \mathrm{erg}$. Because of these high uncertainties, we explore a wide range of parameter values for $E_{\text {jet }}$ and $E_{\text {iso }}$, as reported in Table 2. The other parameters varied are $\eta$, a factor determining the maximum acceleration energy of protons, and $r_{\text {is }}$, which is the internal shock radius. Further details on the model and its parameters are in Appendix A,

The radial density profiles $\delta(r)$ for the PopIII progenitor mass-models, $M=200 M_{\odot}$ and $M=60 M_{\odot}$, have been calculated in (Heger \& Woosley '02) and (Rockefeller et al. '06), respectively. We would like to comment on the apparent contradiction between the $\mathcal{I}_{\text {III }} \propto \delta\left(M_{\text {III }}-300 M_{\odot}\right)$ motivated in the previous section and the $M=200 M_{\odot}$ model used for the calculation for the neutrino flux. Physically, stars with masses 

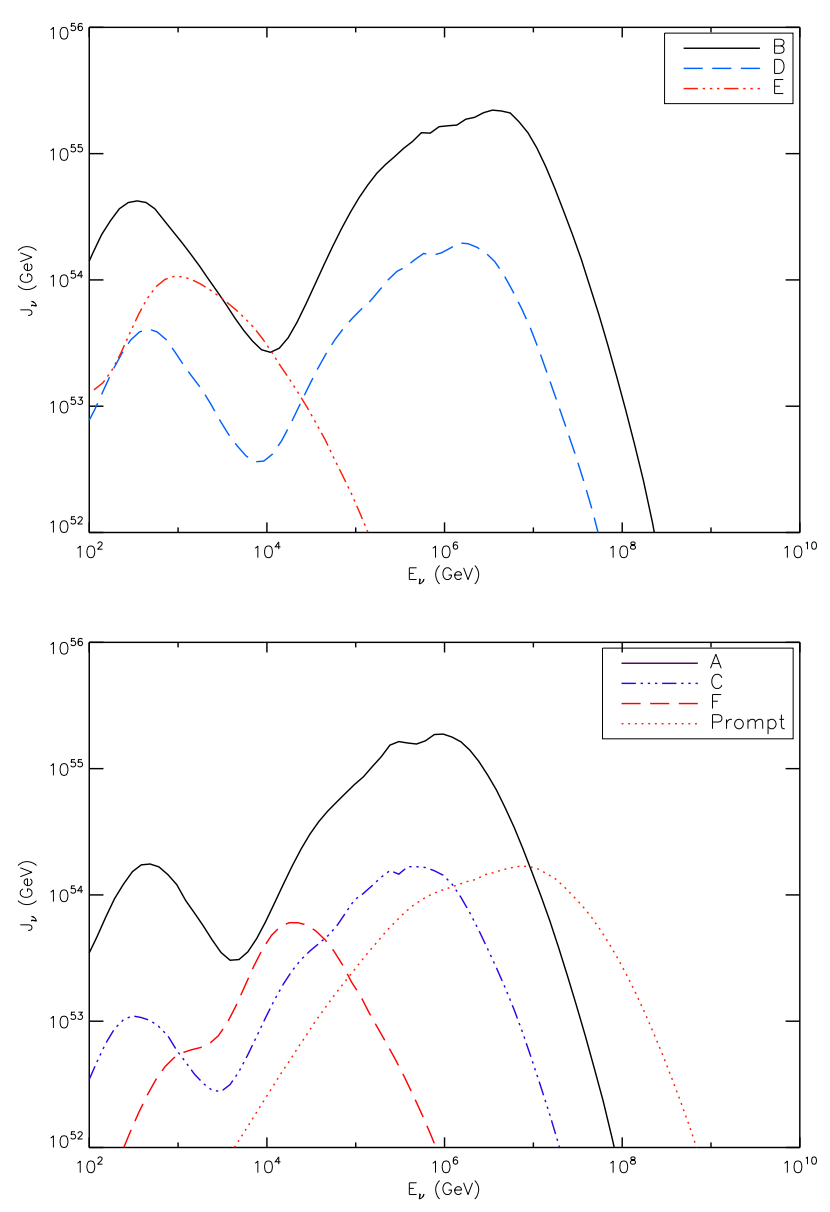

Fig. 1. $-J_{\nu}$ for the different models used, see Tab. 2

$M=140 M_{\odot}<\mathrm{M}<M=260 M_{\odot}$ are known to directly explode as PISNe without the formation of a Black Hole, thus being inefficient GRB progenitors. While important for the GRB rate, however, we expect that the differences in the density profile for the two star masses are much less pronounced in the neutrino yields, and certainly within the uncertainties and assumptions made in such an uneven ground.

In Fig. 1 we show the neutrino emission spectra at the redshift of the source $J_{\nu, \mathrm{III}}(E)$ from the PopIII GRB models considered in Table 2, also compared with the spectrum $J_{\nu, \mathrm{II}}(E)$ for the PopII model Prompt.

In the scenario where internal shocks and termination shocks occur at sufficiently small radii inside the progenitor star, almost accelerated protons are depleted by hadronic and photomeson reactions.

However, produced mesons and muons suffer from cooling processes. The strong magnetic field and copious photon field make pions and muons cooler before they decay. Hence, the neutrino flux will be suppressed due to cooling processes. In Fig. 2, the relative flux level basically reflects the differences among isotropic energies of models. However, the strong field makes mesons and muons give up their energies, so that such suppression becomes important. For example, the difference on the flux level between Model $\mathrm{E}$ and Model $\mathrm{F}$ is reduced. Furthermore, the strong field can force the higher break energy, which is determined by these cooling processes,
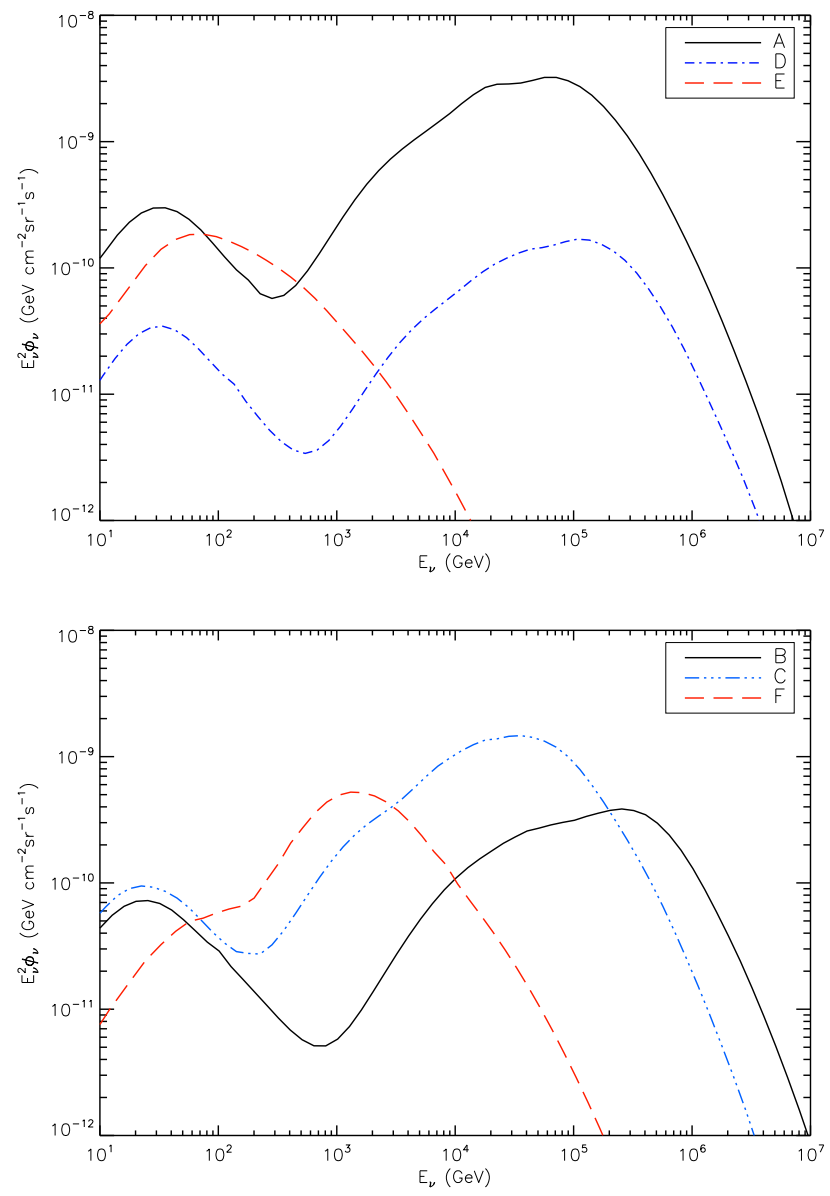

FIG. 2.- The $E_{\nu}^{2} \Phi_{\nu, \text { III }}$ for different $J_{\nu, \text { III }}$ models, assuming $\mathrm{CF} 05_{a}$ as EoR model.

to be shifted to lower energies. This effect also leads to a double peak structure as shown in Fig.1 for Model A-D. The lower peak corresponds to the contribution from the termination shock. In the termination shock, nonthermal protons that are accelerated in the internal shocks interact with thermal photons produced by electrons that are accelerated in the termination shock. On the other hand, the higher is attributed to that from the internal shocks themselves. If we assume the similar equipartion parameters, the termination shock can lead to the stronger field than that of internal shocks. Therefore, neutrinos from this region have lower energies due to pion and muon cooling. Hence, the double peak structures can appear. However, the prominence of this feature is probably due to the simplicity of the model considered. In realistic situations, the emission regions would not be approximated by the simplest two zone model (internal shock region and termination shock region), and the gradient of the field strength should be taken into account. Then, the double peak structure would be more smoothed, although a detailed treatment of this issue clearly goes beyond the scope of this paper.

\section{RESULTS AND DISCUSSION}

In this section we present our results for the neutrino fluxes expected at the Earth. In Fig. 22 we show the contribution of PopIII stars calculated as described in the previous sections, assuming $\mathrm{CF} 05_{a}$ as a fiducial EoR 
model. In general, these fluxes present a huge variability due to the large uncertainties on the production parameters, yet they are all extremely low if compared with what found in (Schneider et al. '05).

In Fig. 3 we compare the high energy neutrino flux at Earth expected from PopII stars with the one from PopIII for different but mutually consistent EoR models, as denoted by the same line-style. When accounting for the assumed baryon loading factor of $\xi_{\text {acc }}=10$ (see Appendix for the role of this parameter) the estimated contribution from PopII stars agrees with typical results found in the literature, and the dependence on the EoR model used is marginal, reflecting the fact that at low redshifts (where most of the signal comes from) all models must agree with the available data ${ }^{1}$. On the other hand, the situation for PopIII is different. The solid and dashed lines assume the model A neutrino yields, which as shown in Fig. 2 maximizes the neutrino production. The dotted line instead follows from the CF06 model, where we have implemented a Prompt spectrum for $J_{\nu \text {,III }}$ coherently with the assumption of a Salpeter IMF. We have treated this case as the PopII one, thus obtaining the fraction of collapsars $\gamma_{\mathrm{III}} \approx 10^{-2}$, consistently with no metallicity evolution beyond $z=4.5$. This illustrates the high energy neutrino flux for the case of an hypothetical low-mass PopIII generation; whereas the CF06 SFR coupled with the A model for $J_{\nu, \text { III }}$ gives results almost indistinguishable from $\mathrm{CF} 05_{b}$ and we have therefore not plotted it. The solid and dashed lines show that the SFR has only a minor effect on the neutrino flux at Earth; on the other hand, their comparison with the dotted line shows that the IMF plays a fundamental role in shaping the flux at Earth, affecting the fraction of stars that will give rise to a collapsar and the magnitude of their explosion energy.

A fairly robust outcome of our study is however that neutrinos from PopIII GRBs will not be detectable with current or near future experiments. In fact, the PopIII flux shown in Fig. 3 falls below both the current AMANDA-II bound (4-years data, see (Halzen '06)) and future prospects for five years of IceCube exposure. Even worse, this contribution is buried beneath the atmospheric neutrinos, whose average spectrum and uncertainty is plotted in Fig 2 according to the compilation recently reported in (Evoli et al '07). Also, it is worth stressing that while the PopII flux we have plotted in Fig 3 is a relatively robust expectation because the background can be evaluated from the observed GRB rate, the PopIII one is an absolute upper limit, as it assumes that all the stellar events will give rise to a collapsar with high energy neutrino emission. These results greatly disagree with the much more optimistic estimates obtained in (Schneider et al. '05), thus motivating some explanation. We believe that our discrepancy with previous results is to impute to the different normalization of PopIII stars SFR. In fact, as it can be easily derived from Fig 1 in (Schneider et al. '05), the neutrino spectra results plotted in their Fig 2 require a rate of $\sim 10^{9}$ GRBs per year. The rate adopted here can be estimated in $\approx 10^{5}$ GRBs per year for $\mathrm{CF} 05_{a}$ model, as reported in more

\footnotetext{
1 The slight discrepancy with previous results in the PopII neutrino flux at Earth is due to the higher GRB rate we use for $3<z$, as it arises from the reionization models we use.
}

details in (Iocco '07). This four orders of magnitude discrepancy accounts for most of the disagreement with our predictions.

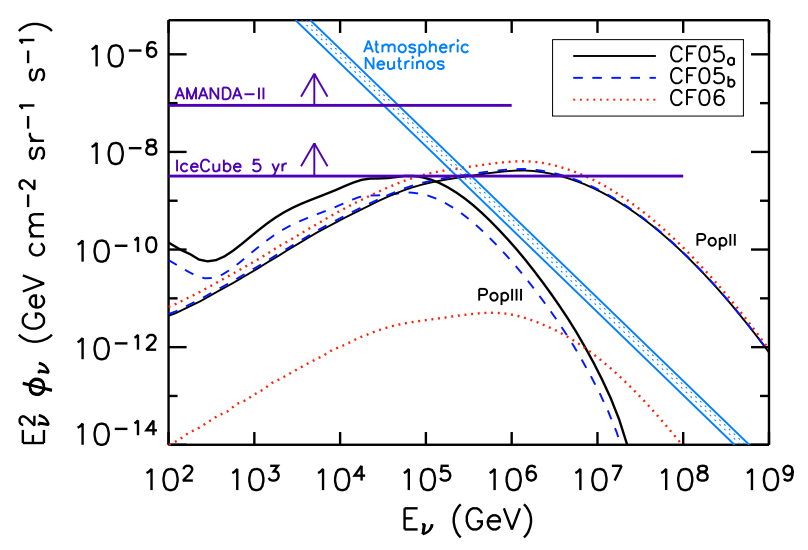

FIG. 3.- The muon neutrino flux $E_{\nu}^{2} \Phi_{\nu}$ for the different Reionization models, using the A model for $J_{\nu, \mathrm{III}}$. Upper-right set of curves refer to the PopII flux, lower-left ones to the PopIII contribution under extreme assumptions (see text).

\section{CONCLUSION}

We have performed a study of the high energy neutrino diffuse background which is to be expected from Population III stars, under the assumption that they will end their lives as GRB. We have compared it with the analogue expected from Population II stars, using mutually consistent SFRs obtained from EoR models available in literature. Our estimate of the PopII GRB rate has been performed under widely accepted assumptions and leads to estimates of the high energy neutrino fluxes which are in agreement with previous results (Murase \& Nagataki '05; Razzaque et al. '03). On the other hand, we have presented a maximal model for PopIII stars, assuming that all of them will end their lives with a GRB, either choked or not. Even under such optimistic assumptions, a detection of the diffuse high energy neutrino background expected from Population III stars appears out of reach. Even worse, the contribution from PopII GRBs would contaminate that from PopIII GRBs. In addition, we cannot expect neutrino signals correlated with gamma-rays from GRBs, because it is thought to be very rare to see PopIII GRBs by the current satellite such as Swift. Hence, the PopIII neutrino signals are expected to be hidden by atmospheric neutrino background. With reasonable values of the nonthermal baryon loading factor, an extreme contribution from PopIII GRBs - including possible choked burstsfalls in fact underneath IceCube five years sensitivity and is overwhelmed, at low energies, by the atmospheric neutrino background. This implies that although PopIII stars may contribute to the high-energy diffuse neutrino background and their spectrum would be indeed sensitive to their IMF and SFR, neutrinos cannot be used as a diagnostic tool to check properties of either the population of stars during the epoch of Reionization or the GRB internal shock properties, thus confirming the elusive nature of the earliest generation of stars. 


\section{ACKNOWLEDGMENTS}

F.I. thanks A. Heger and C.L. Fryer for kindly providing the stellar models, and A. Ferrara for kind clarifications about EoR models. P.S. acknowledges support by the US Department of Energy and by NASA grant NAG5-10842. This work is in part supported by a Grantin-Aid for the 21st Century COE "Center for Diversity and Universality in Physics" from the Ministry of Education, Culture, Sports, Science and Technology of Japan. S.N. is partially supported by Grants-in-Aid for Scientific Research from the Ministry of Education, Culture, Sports, Science and Technology of Japan through No. 19104006 and 19740139 . K.M. is partially supported by Japan Society for the Promotion of Science.

\section{APPENDIX}

\section{MODELING NEUTRINO EMISSION FROM JETS INSIDE THE GRB PROGENITOR STAR}

Here we summarize the basic mechanisms by which neutrinos are produced in jets inside the GRB progenitor star.

We assume that a (long) GRB is a huge stellar collapse leading to the formation of a highly rotating black hole with an accretion disk, during which a globally asymmetric, relativistic jet is launched from the GRB progenitor. The GRB observations imply that the Lorentz factor of jets is very large $\left(\Gamma_{\text {jet }} \sim 100\right)$ in the gamma-ray emitting region. This is the final value which is achieved outside the star, and it may be representative of the intrinsic injection Lorentz factor. Of course, we do not know the intrinsic value and it may also be much smaller than the final value. We shall briefly comment on the latter case later. When the jet propagates inside the star, it will produce a bow shock ahead of it. This jet is capped by a termination shock and a reverse shock. The jet termination radius (where the jet is decelerated) is indicated by $r_{\mathrm{h}}$. If the jet is highly variable and the variability time scale $\delta t$ is small enough, internal shocks can also occur inside the pre-decelerated jet. The internal shock radius is written as $r_{\text {is }} \approx 2 \Gamma_{\text {jet }}^{2} c \delta t$. In the usual internal shock scenario of GRBs, the observed gamma-rays are attributed to internal shocks which occur in the optically thin region outside the progenitor star. Lower $\Gamma_{\text {jet }}$ and/or $\delta t$ lead to smaller radii below the stellar surface, which will be optically thick. Here, we are interested in such opaque, sub-surface internal shocks, and we assume $r_{\text {is }} \lesssim r_{h}<r_{*}$, where $r_{*}$ is the radius of the progenitor's stellar surface.

These internal shocks are expected to be collisionless, so that both electrons and protons may be accelerated. If the magnetic field is strong enough, electrons can be accelerated up to high energies and radiate photons. The photon density in the pre-decelerated jet is given by

$$
U_{\gamma}^{\mathrm{jet}}=\frac{E_{\mathrm{sh}}}{4 \pi r_{\mathrm{is}}^{2} \Gamma_{\mathrm{jet}} \Delta l},
$$

where $\Delta l$ is the width of subshells, for which we use $\Delta l \approx r / \Gamma_{\text {jet }}$ because we have assumed that the jet acceleration has already ceased. The magnetic energy density in the field $B$ is expressed as

$$
U_{B}^{\text {jet }}=\frac{B^{2}}{8 \pi}=\xi_{B}^{\text {jet }} U_{\gamma}^{\text {jet }}
$$

Due to the existence of the strong magnetic field, electrons radiate synchrotron photons. These photons will be thermalized, if the jet is optically thick to Thomson scattering. We assume $\xi_{B}^{\text {jet }}=0.1$ and expect that the jet is opaque. Therefore, we can approximate the spectral distribution of the radiation of energy density $U_{\gamma}^{\text {jet }}$ by a blackbody spectrum, and associate to it a temperature $T_{\text {jet }}$. The above approximate treatment is accurate enough for our purpose.

Protons get accelerated in the shocks as well. It is widely believed that cosmic rays can be accelerated by the firstorder Fermi acceleration mechanism. We assume that this mechanism can work efficiently and adopt the spectral index $\sim 2$. The non-thermal proton energy density is expressed as $U_{p}^{\text {jet }}=\xi_{\text {acc }} U_{\gamma}^{\text {jet }}$, where $\xi_{\text {acc }}$ is the nonthermal baryon loading factor and for efficient proton acceleration we can express as $\xi_{\text {acc }} \sim 1 / \epsilon_{e}, \epsilon_{e}$ being the fraction of internal energy carried by the electrons. Because plausible values of this parameter is not known yet, we adopt $\xi_{\text {acc }}=10$, which corresponds to the assumption that the energy of protons per logarithmic energy bin is comparable to the GRB radiation energy (Waxman \& Bahcall '97; Murase \& Nagataki '05). Too large value of $\xi_{\text {acc }}$ requires too small values of $\epsilon_{e}$, which is usually unexpected in GRBs (but there is no proof because we do not know the total explosion energy). The non-thermal proton spectrum is given by

$$
\frac{d n_{p}^{\text {jet }}}{d \varepsilon_{p}}=\frac{U_{p}^{\text {jet }}}{\ln \left(\varepsilon_{p}^{\max } / \varepsilon_{p}^{\min }\right)} \varepsilon_{p}^{-2}
$$

The minimum energy $\varepsilon_{p}^{\min }$ is set to $10 \mathrm{GeV}$. The choice of this value is not so sensitive to our final results. On the other hand, the maximum energy is important for the purpose of knowing neutrino spectra at the highest energies. The maximum energy is determined by the condition

$$
\frac{e B c}{\eta \varepsilon_{p}^{\max }}=t_{\mathrm{acc}}^{-1} \simeq \sum_{i} t_{i}^{-1}
$$

where at the l.h.s. it appears the Larmor radius of the proton times the pre-factor $\eta(=\mathcal{O}(1-10))$ which depends on the details of acceleration mechanism; $t_{\mathrm{acc}}$ is essentially the acceleration time in the Bohm limit, and the sum at the r.h.s. extends over all the energy loss channels timescales $t_{i}$. 
Accelerated protons can interact with protons themselves or photons, which lead to meson production such as pions and kaons. In this paper, we consider neutrinos produced through photomeson production only. The method of calculation is described in (Murase \& Nagataki '05; Murase '07). Here, we briefly sketch this process by simple analytic considerations. The photomeson production is a threshold process, with a threshold energy of about 145 $\mathrm{MeV}$ in the rest frame of the incident proton. The dominant inelastic channel is $p \gamma \rightarrow \Delta^{+}$with the cross section $\sigma_{p \gamma} \approx 5 \times 10^{-28} \mathrm{~cm}^{2}$ and the inelasticity $\kappa_{p} \approx 0.2$, which is called $\Delta$-resonance. For the cases we consider, the $p \gamma$ optical depth at the $\Delta$-resonance is very large, so that the photomeson production efficiency can also be high. This means that almost all the protons that have sufficiently high energies (above the threshold energy) will be depleted due to photomeson production.

The threshold for $p p$ inelastic interactions is lower, so these processes will also occur. Although this may be an important neutrino source in the $\mathrm{TeV}$ energies (in particular for low Lorentz factors $\Gamma_{\text {jet }}$ ), in this paper we focus on high energy neutrinos produced by sufficiently high energy protons above the threshold energy for photomeson production. We take into account the $p p$ process only for estimating the proton energy loss time scale, treating this process analytically for simplicity.

Next, we consider the interaction between the jet and the progenitor star. As the jet advances through the star, it drives a bow shock ahead of it. The jet is capped by a forward shock, and a reverse shock moves back into the jet, where the relativistic jet is decelerated. The shocked jet plasma and shocked stellar plasma would advance together with a jet head Lorentz factor $\Gamma_{\mathrm{h}} \ll \Gamma_{\text {jet }}$. By equating the pressures behind the forward shock and reverse shocks, one finds the following estimate for the Lorentz factor of the jet head,

$$
\Gamma_{\mathrm{h}} \simeq \Gamma_{\text {jet }}^{1 / 2}\left(\frac{m_{p} n_{p}^{\mathrm{jet}}}{4 \delta}\right)^{1 / 4}
$$

where $m_{p}$ is the proton mass and $\delta$ is the mass density of the environment.

The relative Lorentz factor between the shocked jet plasma and un-shocked jet plasma is

$$
\Gamma^{\prime} \simeq \frac{1}{2}\left(\frac{\Gamma_{\text {jet }}}{\Gamma_{\mathrm{h}}}+\frac{\Gamma_{\mathrm{h}}}{\Gamma_{\mathrm{jet}}}\right) .
$$

Electrons will be accelerated in these shocks. However, the electrons would give up all their energy on a very short time scale, by synchrotron and inverse-compton (IC) cooling, converting a large fraction of the shocked plasma internal energy into radiation. These radiated photons in the shocked jet plasma will be thermalized due to large optical thickness. Hence, target photon density will be approximately a black-body radiation, with an overall energy density

$$
U_{\gamma}^{\mathrm{h}} \simeq\left(4 \Gamma^{\prime}+3\right)\left(\Gamma^{\prime}-1\right) n_{p}^{\mathrm{jet}} m_{p} c^{2}
$$

with an associated temperature $T_{\mathrm{h}}$.

The reverse shock is likely to become radiation dominated, so that the IC cooling by the electrons becomes important and affects the dissipation of jet kinetic energy. If the reverse shock is indeed radiation dominated, the shock thickness would also be of order the mean-free path of thermal photons propagating into the jet. The copious photon field also affects the neutrino spectrum. Charged mesons produced via $p \gamma$ (and $p p$ ) interactions will suffer IC and synchrotron losses, and neutrino spectra will be suppressed. The magnetic energy density in the shocked jet frame is expressed as

$$
U_{B}^{\mathrm{h}}=\xi_{B}^{\mathrm{h}} U_{\gamma}^{\mathrm{h}}
$$

For $\xi_{B}^{\mathrm{h}}=\mathcal{O}(0.1-1)$, the magnetic field strength is very large, up to $\sim 10^{7-9} \mathrm{G}$. Hence, charged particles would suffer from synchroron loss, and one should take into account synchrotron losses of pions in order to calculate neutrino spectra, as we do in our computation.

If non-thermal protons that are accelerated in internal shocks are not completely depleted, they can enter the shocked jet. Such protons are expected to interact with photons in the shocked jet region, and to produce neutrinos. In principle, protons can be accelerated at the termination shocks, too. However, cooling processes such as IC loss significantly will reduce the maximum proton energy there. Hence, we treat the contribution from protons that are accelerated in internal shocks only. The proton spectrum in the frame of the shocked jet plasma is given by

$$
\frac{d n_{p}^{\mathrm{h}}}{d \varepsilon_{p}} \simeq\left(1-f_{p}^{\mathrm{jet}}\right) \Gamma^{\prime 2} \frac{d n_{p}^{\mathrm{jet}}}{d \varepsilon_{p}}
$$

where $f_{p}^{\text {jet }}$ is a fraction of depleted protons in the internal shocks, which can be estimated as the ratio of the dynamical timescale involved with the typical energy loss time. As protons approach the reverse shock, they can interact with photons via photomeson production, if proton energy is above the threshold for photomeson production. It turns out that also in this region the photon density is high enough to ensure that almost all protons that have sufficiently high energies are depleted due to photomeson production. However, neutrino energy from this region is smaller than that from the pre-decelerated jet.

The $p p$ interaction also occurs in the shocked jet plasma, and a further neutrino source is due to non-thermal proton interactions with cold protons in the star after they escape the shocked jet plasma. For more detailed predictions, 
these processes should be taken into account. Yet, given the high efficiencies of the photohadronic processes for our typical parameters, we expect them to be at most subleading at the high energies we are mostly interested in.

Finally, note that synchrotron, IC, and adiabatic cooling of pions and muons are important in shaping our final result. The treatment of these cooling processes is also similar to that used in (Murase \& Nagataki '05; Murase \& Nagataki '06). Analytic considerations on effects of these cooling processes can be found in (Razzaque et al. '03).

\section{REFERENCES}

T. Abel, G. L. Bryan and M. L. Norman, Science 295, 93 (2002) astro-ph/0112088.

J. Ahrens et al. [IceCube Collaboration], Astropart. Phys. 20, 507 (2004) arXiv:astro-ph/0305196.

A. Amati et al., Astron. Astrophys. 390, 81 (2002) astro-ph/0205230.

T. R. Choudhury and A. Ferrara, Mon. Not. Roy. Astron. Soc. 361, 577 (2005) astro-ph/0411027.

T. Roy Choudhury and A. Ferrara, Mon. Rot. R. Astron. Soc. 371 , L55 (2006) astro-ph/0603617.

C. Evoli, D. Grasso and L. Maccione, arXiv:astro-ph/0701856

E. E. Fenimore and E. Ramirez-Ruiz, astro-ph/0004176 .

D. A. Frail et al. Astrophys. J. 562, L55 (2001) astro-ph/0405602

G. Ghirlanda, G. Ghisellini, D. Lazzati Astrophys. J. 616, 331 (2004) astro-ph/0102282.

F. Halzen, astro-ph/0611915

A. Heger, N. Langer and S. E. Woosley, Astrophys. Journ. 528, 368 (2000) astro-ph/9904132.

A. Heger and S. E. Woosley, Astrophys. J. 567, 532 (2002) astro-ph/0107037.

A. Heger, C. L. Fryer, S. E. Woosley, N. Langer and D. H. Hartmann, Astrophys. Journ. 591, 288 (2003) astro-ph/0212469.

F. Iocco, the Proceedings of the "First GLAST Symposium", Stanford, Palo Alto, 5-8 Feb 2007.

F. Iocco, G. Mangano, G. Miele, G. G. Raffelt and P. D. Serpico, Astropart. Phys. 23, 303 (2005) astro-ph/0411545.

F. Iocco, G. Mangano, G. Miele, O. Pisanti and P. D. Serpico, Phys. Rev. D 75, 087304 (2007) astro-ph/0702090

A. Kashlinsky, R. G. Arendt, J. C. Mather and S. H. Moseley, Nature 438, 45 (2005) astro-ph/0511105.

P. Kroupa, Mon. Not. Roy. Astron. Soc. 322, 231 (2001) astro-ph/0009005.
A. I. MacFadyen, S. E. Woosley and A. Heger, Astrophys. J. 550, 410 (2001) astro-ph/9910034.

A. Maeder and G. Meynet, Astron. Astrophys. 361, 101 (2000) astro-ph/0006404.

P. Meszaros and E. Waxman, Phys. Rev. Lett. 87, 171102 (2001) astro-ph/0103275.

T. Murakami, Y. Yonetoku, M. Umemura, T. Matsubayashi and R. Yamazaki, Astroph. Journ. 625, L13 (2005) astro-ph/0504347.

E. Waxman and J. Bahcall, Phys. Rev. Lett. 78, 2292 (1997)

K. Murase and S. Nagataki, Phys. Rev. D 73, 063002 (2006) astro-ph/0512275].

K. Murase and S. Nagataki, Phys. Rev. Lett. 97, 051101 (2006)

K. Murase, in preparation

Achterberg et al., astro-ph/0702265.

F. Nakamura and M. Umemura, Astrophys. J. 548, 19 (2001) astro-ph/0010464.

B. W. O'Shea, T. Abel, D. Whalen and M. L. Norman, Astrophys. J. 628, L5 (2005) astro-ph/0503330.

S. Razzaque, P. Mész'aros and E. Waxaman Phys. Rev. D 68, 083001 (2003).

G. Rockefeller, C. L. Fryer and H. Li, astro-ph/0608028.

R. Schneider, D. Guetta and A. Ferrara, Mon. Not. Roy. Astron. Soc. 334, 173 (2002) astro-ph/0201342.

D. Spergel et al., astro-ph/0603449.

D. Yonetoku, T. Murakami, T. Nakamura, R. Yamazaki, A. K. Inoue and K. Ioka, astro-ph/0309217.

S. C. Yoon, N. Langer and C. Norman, Astron. Astrophys. 460 , 199 (2006) astro-ph/0606637.

H. Yuksel and M. D. Kistler, Phys. Rev. D 75, 083004 (2007) astro-ph/0610481. 